\title{
Study of Lamb Waves for Non-Destructive Testing Behind Screens
}

\author{
P. Kauffmann, M.-A. Ploix, J.-F. Chaix, C. Gueudré, G. Corneloup and F. Baqué
}

\begin{abstract}
The inspection and control of sodium-cooled fast reactors (SFR) is a major issue for the nuclear industry. Ultrasonic solutions are under study because of the opacity of liquid sodium. In this paper, the use of leaky Lamb waves is considered for non-destructive testing (NDT) on parallel and immersed structures assimilated as plates. The first phase of our approach involved studying the propagation properties of leaky Lamb waves. Equations that model the propagation of Lamb waves in an immersed plate were solved numerically. The phase velocity can be experimentally measured using a two dimensional Fourier transform. The group velocity can be experimentally measured using a short-time Fourier transform technique. Attenuation of leaky Lamb waves is mostly due to the re-emission of energy into the surrounding fluid, and it can be measured by these two techniques.
\end{abstract}

Index Terms-Group velocity, Leaky Lamb waves, NonDestructive Testing (NDT), Phase velocity, Re-emission, Sodium Fast Reactor (SFR), Ultrasound.

\section{INTRODUCTION}

$\mathrm{T}_{\mathrm{b}}$ he sodium-cooled fast reactor (SFR) technology has been chosen for the French $4^{\text {th }}$ generation of nuclear power plants. With liquid sodium as coolant, the improvement of in-service inspection and repair (ISI\&R) techniques has been identified as a major issue for the ASTRID project (Advanced Sodium Technological Reactor for Industrial Demonstration) [1], [2]. Ultrasonic solutions are being studied in order to inspect the main vessel and different parts of ASTRID. Fig. 1 shows different inspection strategies with ultrasonic transducers inside and outside the main vessel. Immersion of specific ultrasonic transducers in liquid sodium is under study [3], [4] (1 $1^{\text {st }}$ strategy in Fig. 1). However, inspections using a transducer located outside the main vessel

Manuscript submitted June 1, 2017. This work was supported by the CEA/ Cadarache, France.

P. Kauffmann, M.-A. Ploix, J.-F. Chaix, C. Gueudré, and G. Corneloup are with Aix Marseille Univ, CNRS, Centrale Marseille, LMA, Laboratoire de Mécanique et d'Acoustique, Equipe Ondes et Imagerie, site d'Aix en Provence, Marseille, France (e-mail: pierre.kauffmann@cea.fr).

P. Kauffmann and F. Baqué are with the CEA Cadarache DEN/DTN/STCP/LIET (Laboratoire d'Instrumentation et d'Essais Technologiques), Saint Paul Lez Durance, France (e-mail: francois.baque@cea.fr).

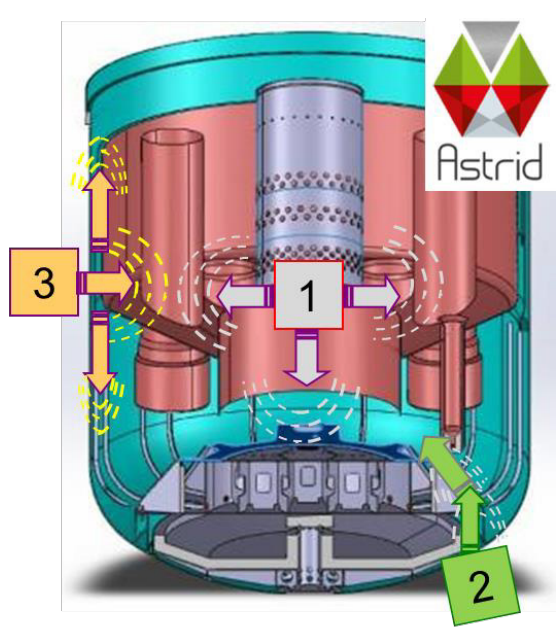

Fig. 1. Different ultrasonic inspections strategies for ASTRID: inspection with a transducer immersed in sodium ( ${ }^{\text {st }}$ strategy), with a transducer in the inter-vessel gap using immersed structures as a wave guide ( $2^{\text {nd }}$ strategy), and through the main vessel ( $3^{\text {rd }}$ strategy)

(in the inter-vessel gap filled with gas) have shown promising potential [5], [6]. The second strategy indicated in Fig. 1 is also under study [7]. We will focus on the third strategy indicated in Fig. 1.

The objective is to not only inspect the main vessel, but also the structures behind it. This includes the redan (the component separating the hot and the cold pool), the internal baffles, sodium pump, and the heat exchanger skirts. The diameter of the main vessel and internal baffles is quite large (about $16 \mathrm{~m}$ ) and justify to approximate locally their shape by two parallel and immersed plates (Fig. 2). The challenge is then to propagate ultrasonic waves from one plate to another and to control them.

Mechanical waves that propagate in an elastic plate are called Lamb waves [8]. Their use for NDT is widely studied in the case of plates in vacuum (or in air) [9], [10]. However, the configuration of several parallel and immersed plates is quite innovative. References are rare, even if an artificial notch in the second and third plate has been observed experimentally under certain conditions [5], [6]. We can also cite [11]: it has been shown that for a system of two semi-immersed plates, Lamb waves propagate from the first plate to the second.

As a first step, this paper describes the propagation and reemission of Lamb waves in the case of an immersed plate. Equations used to calculate the phase velocity, group velocity and attenuation are specified. Experiments in water with two different data processing techniques (2D Fourier transform and short-time Fourier transform) are briefly presented. 
Finally, the experimental results are discussed.

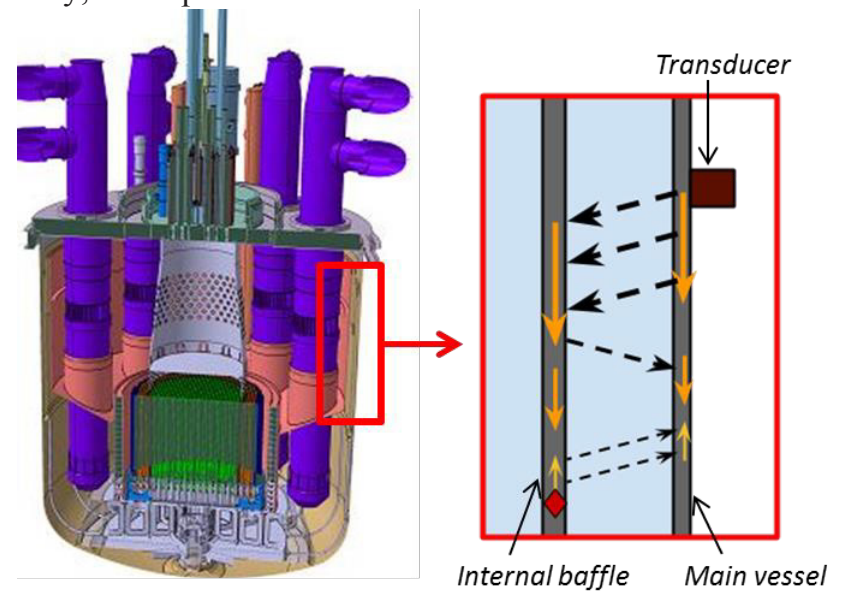

Fig. 2. Cross-section of the ASTRID reactor block with location of the area to be inspected (red box) and a schematic view of this area with the expected ultrasonic path

\section{Propagation AND ATtenuation OF LAMB WAVES}

Two types of Lamb modes can be differentiated: symmetric and antisymmetric, which are designated with the letters $\mathrm{S}$ and A respectively. Symmetric (and antisymmetric respectively) For an elastic plate of thickness $h$ end density $\rho$ immersed in a fluid of density $\rho_{0}$, propagation equations can be found in [12]. These equations can be solved numerically, and it allows us to determine the phase velocity $c_{p}$ (Fig. 3) and attenuation $k_{2}$ (Fig. 4) of the Lamb modes.

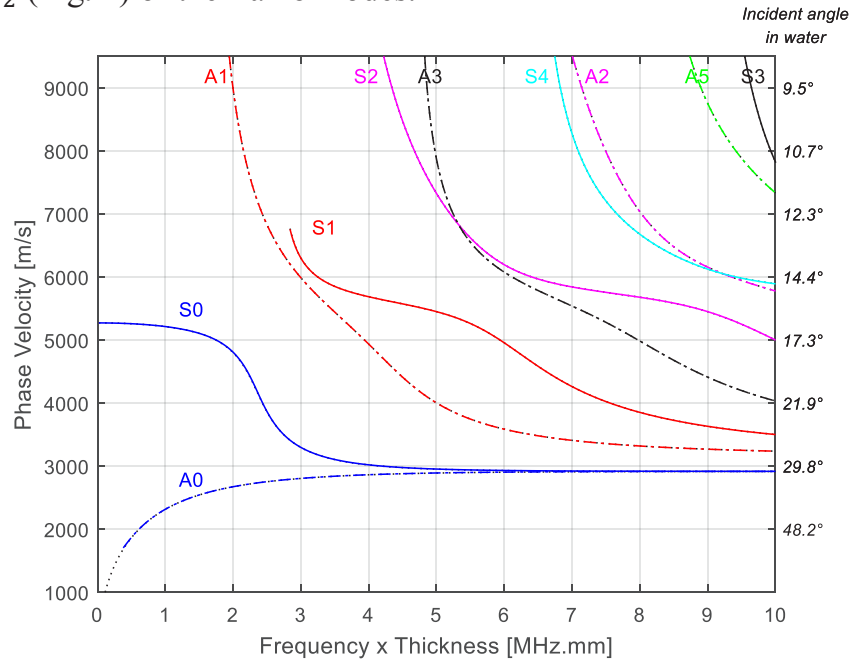

Fig. 3. Phase velocity of Lamb modes for a stainless steel plate $(h=7.8 \mathrm{~mm}$, $\rho=7950 \mathrm{~kg} / \mathrm{m}^{3}, \quad c_{L}=5750 \mathrm{~m} / \mathrm{s}$ and $c_{T}=3150 \mathrm{~m} / \mathrm{s}$ ) immersed in water. Results for a plate in vacuum are also plotted but are too similar to be distinguished from the immersed case

Fig. 3 shows that the phase velocity of the Lamb modes is strongly dependent on the frequency. This dispersive propagation has two major consequences. First of all, short time wave packets will spread in space and time as they propagate. Therefore, the pulse duration increases with propagation, and the maximum amplitude measured decreases because of this spreading. Secondly, the velocity of the wave packet is no longer the phase velocity, but the group velocity.
The group velocity can be calculated from the phase velocity [13].

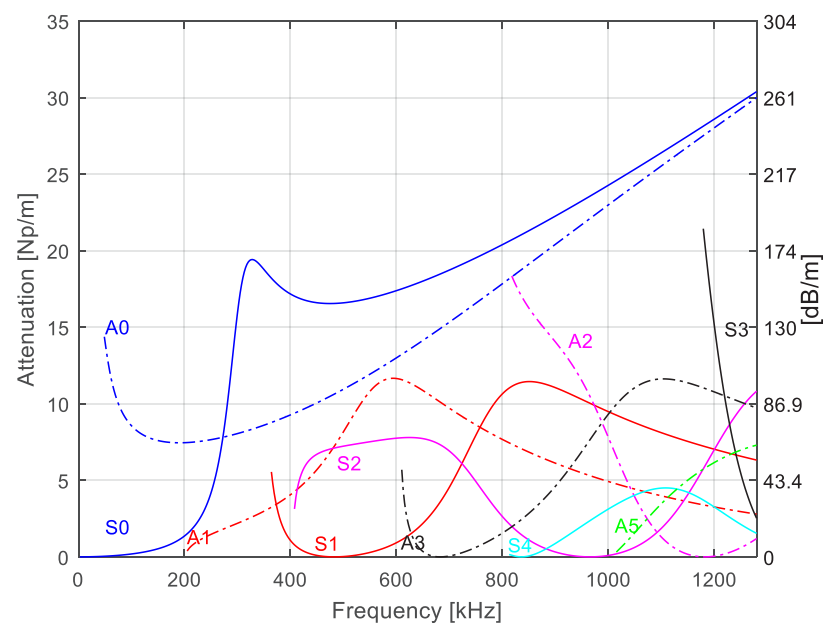

Fig. 4. Attenuation coefficient $k_{2}$ computed with the same parameters as in Fig. 3. The $x$-axis now represents the frequency and no longer the product frequency thickness because attenuation is strongly dependent on the thickness. The results given are for a plate that was $7.8 \mathrm{~mm}$ thick.

For more information, the effects of dispersive propagation on a pulse are studied in detail in reference [14].

Phase velocity, group velocity and leaky attenuation are key characteristics to performing NDT behind screens. We will present the experimental set-up that we used to measure these characteristics in the following section.

\section{EXPERIMENTATION IN WATER AND DATA PROCESSING}

Since experiments in liquid sodium are complex and liquid sodium is quite similar to water from an acoustic viewpoint, experimental measurements and observations were performed in water at room temperature.

As shown in Fig. 5, we considered a single plate immersed in water. A transducer was used to send a pulse at a given central frequency and a chosen incident angle $\theta_{i}$. The incident angle was chosen on the basis of the Snell-Descartes law (1) in order to excite the phase velocity of a chosen Lamb mode in the plate. Some of the incident angles in water are shown on the right scale in Fig. 3.

$$
\sin \left(\theta_{i}\right)=\frac{c_{w}}{c_{p}}
$$

with $c_{w}$ the speed of sound in water, $c_{p}$ the phase velocity of the chosen Lamb mode, and $\theta_{i}$ the incident angle to the normal of the plate.

However, this technique may excite more than one Lamb mode if their respective phase velocities are close enough regardless the spreading angle of the beam [15].

In order to separate the contribution of each mode, we used a 2D Fourier transform method [16]. Wave propagation was no longer integrated into the \{time; position\} domain, but into the \{frequency; wave number\} domain.

Moreover, the attenuation was measured by reproducing the 
2D Fourier transform twice in two different propagation zones. This technique is presented in [17], but is applied now to measure the leaky attenuation and not anymore the attenuation of composite material.

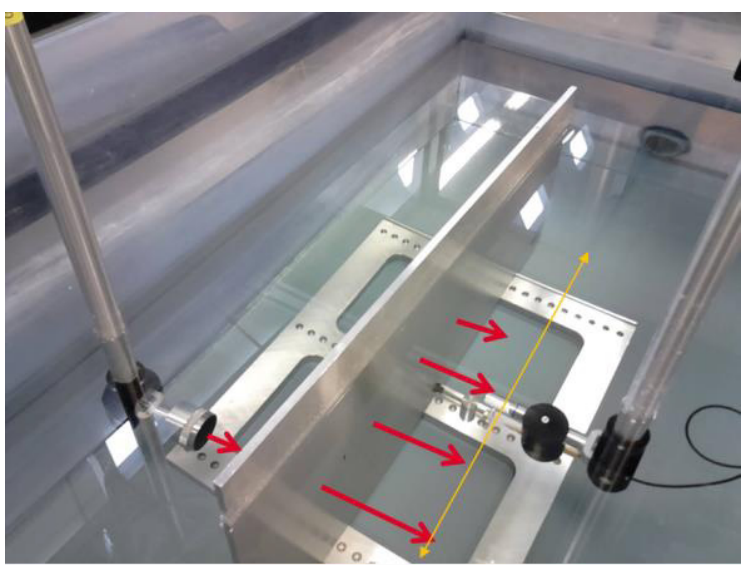

Fig. 5. Experimental bench: a transducer sends bulk waves at a chosen incident angle $\theta_{i}$ onto a plate $7.8 \mathrm{~mm}$ thick, immersed in water. A needle hydrophone scans the transmitted field on a line parallel to the plate. Bolds arrows symbolize the waves in water (incident and re-emitted waves). The thin arrow symbolizes hydrophone displacement

In the literature, group velocity are generally measured by measuring the speed of the envelope [14], [18]. In the case of an immersed plate, however, attenuation may be too strong and multi-modal propagation is likely to append, preventing the measurement of velocities on the raw data. This is why we chose to use a time frequency technique to separate the contribution of each mode in the frequency range. This technique is detailed in [19] but is applied now to the case of an immersed plate and not anymore to a plate in air.

In addition to the ultrasonic transducer used in Fig. 5, two other transducers with different central frequencies were used around $110 \mathrm{kHz}$ (i.e. $0.9 \mathrm{MHz} . \mathrm{mm}$ ) and $250 \mathrm{kHz}$ (i.e. 1.95 MHz.mm). For each transducer, the experimentation was conducted for two or three different incidence angles in order to study the various Lamb modes.

\section{CONCLUSION}

We have shown that techniques used in the literature to measure the propagation of Lamb waves can be applied for an immersed plate. Then the study of the leaky attenuation becomes possible. This will enable us to understand and predict the behavior of an ultrasonic wave in a set of several parallel and immersed plates. It is a primordial step to performing NDT behind screens.

Further study will focus on the propagation of Lamb waves in a set of parallel plates, including finite-element simulations and experimentation in water. The reflection on a defect will also be studied.

\section{REFERENCES}

[1] F. Jadot, F. Baqué, J. P. Jeannot, G. de Dinechin, J. M. Augem, et J. Sibilo, «ASTRID sodium cooled fast reactor: Program for improving in service inspection and repair », in 2011 2nd International Conference on Advancements in Nuclear Instrumentation, Measurement Methods and their Applications (ANIMMA), 2011, p. 1-8.

[2] F. Baqué, F. Jadot, R. Marlier, J. F. Saillant, et V. Delalande, « In Service Inspection and Repair of the sodium cooled ASTRID reactor prototype ", in Proceedings of ICAPP'15, Nice (France), 2015.

[3] F. Baqué, F. Reverdy, J.-M. Augem, et J. Sibilo, « Development of Tools, Instrumentation and Codes for Improving Periodic Examination and Repair of SFRs », Sci. Technol. Nucl. Install., n ${ }^{\circ} 718034$, p. 19, 2012.

[4] F. Baqué, F. Jadot, R. Marlier, J. F. Saillant, et V. Delalande, « In Service Inspection and Repair of Sodium cooled ASTRID Prototype ", in 2015 4th International Conference on Advancements in Nuclear Instrumentation Measurement Methods and their Applications (ANIMMA), 2015.

[5] G. Corneloup, M.-A. Ploix, J.-F. Chaix, I. Lillamand, et F. Baqué, « Potential of Ultrasounds for NDT of a structure located behind parallel immersed plates ", in Review of progress in Quantitative

NonDestructive Evaluation: volume 30 A; volume $30 B$, 2011, vol. 1335 , p. 1695-1700.

[6] F. Baqué, K. Paumel, G. Corneloup, M. A. Ploix, et J. M. Augem, « Non destructive examination of immersed structures within liquid sodium », in 2011 2nd International Conference on Advancements in Nuclear Instrumentation, Measurement Methods and their Applications (ANIMMA), 2011, p. 1-5.

[7] Q. Elie, F. L. Bourdais, K. Jezzine, et V. Baronian, « Study of guided wave transmission through complex junction in sodium cooled reactor », in 2015 4th International Conference on Advancements in Nuclear Instrumentation Measurement Methods and their Applications (ANIMMA), 2015, p. 1-5.

[8] H. Lamb, « On Waves in an Elastic Plate », Proc. R. Soc. Lond. Math. Phys. Eng. Sci., vol. 93, nº 648, p. 114-128, mars 1917.

[9] I. A. Viktorov, Rayleigh and Lamb Waves: Physical Theory and Applications, Softcover reprint of the original 1st ed. 1967 edition. Springer, 2013.

[10] P. D. Wilcox, M. J. S. Lowe, et P. Cawley, « Mode and transducer selection for long range lamb wave inspection », J. Intell. Mater. Syst. Struct., vol. 12, nº 8, p. 553-565, 2001.

[11] M. Schmitt et al., « Detection of coatings within liquid-filled tubes and containers by mode conversion of leaky Lamb waves », J. Sens. Sens. Syst., vol. 2, n 1, p. 73-84, mai 2013.

[12] L. G. Merkulov, « Damping of normal modes in a plate immersed in a liquid », Sov Phys Acoust, vol. 10, n 2, p. 169-173, 1964.

[13] J. L. Rose, Ultrasonic Waves in Solid Media. Cambridge University Press, 1999.

[14] P. Wilcox, M. Lowe, et P. Cawley, « The effect of dispersion on longrange inspection using ultrasonic guided waves ", NDT E Int., vol. 34, $\mathrm{n}^{\mathrm{o}}$ 1, p. 1-9, janv. 2001.

[15] T. D. K. Ngoc et W. G. Mayer, « A General Description of Ultrasonic Nonspecular Reflection and Transmission Effects for Layered Media », IEEE Trans. Sonics Ultrason., vol. 27, nº 5, p. 229-235, sept. 1980.

[16] D. Alleyne et P. Cawley, « A two-dimensional Fourier transform method for the measurement of propagating multimode signals », $J$. Acoust. Soc. Am., vol. 89, no 3, p. 1159-1168, mars 1991.

[17] M. Castaings, C. Bacon, B. Hosten, et M. V. Predoi, « Finite element predictions for the dynamic response of thermo-viscoelastic material structures ", J. Acoust. Soc. Am., vol. 115, n 3, p. 1125-1133, mars 2004.

[18] X. Luis Dean-Ben, C. Trillo, A. F. Doval, et J. L. Fernandez, « Phase and group velocity measurement of ultrasonic guided wavetrains in plates by pulsed TV holography », J. Acoust. Soc. Am., vol. 127, n 4, p. 2210-2219, avr. 2010.

[19] M. Niethammer, L. J. Jacobs, J. Qu, et J. Jarzynski, « Time-frequency representation of Lamb waves using the reassigned spectrogram », $J$. Acoust. Soc. Am., vol. 107, n 5, p. L19-L24, mai 2000. 